\title{
Immunocytochemical examination of bone marrow in disseminated neuroblastoma
}

\author{
P J Carey, L Thomas, G Buckle, M M Reid
}

\begin{abstract}
To assess the usefulness of immunocytochemical analysis of bone marrow in patients with neuroblastoma, marrow smears from 33 staging procedures in 12 patients were examined using an indirect immunoalkaline phosphatase technique with monoclonal antibodies raised against human neural tissue. Marrow aspirate and trephine collagenase digest specimens from individual sites were each tested with the monoclonal antibody UJ13A and with a pool of three related antibodies. The results were compared with morphological assessment of conventionally stained aspirates and trephine specimens taken at the same time. Immunostaining suggested the presence of tumour in seven of 18 staging procedures in which conventional techniques had shown infiltration. Tumour infiltration was also suggested in four of 10 staging procedures with suspicious trephine specimens, but in none of three with relatively innocent histological and cytological features.

Immunological investigation provides no additional information about the presence of infiltration if conventional microscopy has shown definite tumour. When histological appearances are suspicious, immunostaining of stored aspirate smears or collagenase digest specimens may provide evidence of infiltration. There are insufficient data to comment on the value of immunostaining when conventional techniques reveal "normal" marrow, but the impression gained from this study is that immunostaining has a limited role in the detection of metastatic neuroblastoma, which yet remains to be defined.
\end{abstract}

Bone marrow infiltration in disseminated neuroblastoma may be difficult to detect, especially in aspirates, and particularly after treatment has started. Immunological methods for examining marrow with monoclonal antibodies directed against neuroectodermal cell antigens may complement morphological examination of aspirated material.

A previous report described the histological appearances of bone marrow trephine biopsy specimens in this disease and suggested a scheme for grading specimens. ${ }^{1}$ In addition to specimens in which tumour was easily identified, there were specimens with distorted architecture and increased fibrosis which, it was suggested, implied that neuroblastoma had not been eradicated.

This report describes our experience with an indirect immunoalkaline phosphatase technique $^{2}$ applied to a series of staging marrow samples taken from children with stage IV neuroblastoma, and the comparison of results with the morphological appearances of the bone marrow biopsy specimens and the standard Romanowsky stained smears.

\section{Patients and methods}

Twelve children with neuroblastoma who had bone marrow infiltration at diagnosis were studied. All were treated with aggressive chemotherapy. Marrow aspirates and biopsy specimens were obtained from two to four iliac crests on one to six occasions from each child. Thirty three staging procedures were available for analysis.

One set of native bone marrow smears from each child was examined after standard Romanowsky staining. Duplicate sets were wrapped in plastic film and stored at $-20^{\circ} \mathrm{C}$. Trephine biopsy cores were processed for routine histopathological examination, after, in some cases, removal of a fragment for digestion with collagenase, followed by cytocentrifuge preparation of slides of the liberated cells, ${ }^{3}$ which were also wrapped and frozen. Later, slides were thawed, unwrapped, number coded and stained in batches using an indirect immunoalkaline phosphatase method with a labelled second antibody. ${ }^{2}$

Two tests were applied to available smears and collagenase preparations from each iliac crest. One was the monoclonal antibody UJ13A, and the other a "pool" of three antibodies: UJ127.11, UJ181.4, and 5.1.H11. All detect antigens present on cells of neuroectodermal origin and were kindly supplied by Dr J Kemshead, Imperial Cancer Research Fund Laboratories, Institute of Child Health, London. The tumour cells from all 12 children reacted with UJ13A at diagnosis.

Tests with UJ13A and with the "pool" of antibodies were performed either on separate slides or together on different areas of the same slide, depending on the size of the smear available.

The entire surface of each smear was examined independently by two observers (MMR and PC). Results were recorded as positive if immunostaining cells, either singly or in clumps, were thought to represent tumour. A negative result was recorded if the 
Table 1 Immunocytochemical staining of marrow smears compared with marrow aspirate cytology and trephine biopsy histology

\begin{tabular}{|c|c|c|c|c|c|c|c|}
\hline \multirow{3}{*}{$\begin{array}{l}\text { Trephine } \\
\text { histological } \\
\text { grade }\end{array}$} & \multirow{2}{*}{\multicolumn{2}{|c|}{$\begin{array}{l}\text { Aspirate } \\
\text { Romanowsky } \\
\text { smears }\end{array}$}} & \multicolumn{4}{|c|}{ Immunocytochemistry ${ }^{\star}$} & \multirow{3}{*}{$\begin{array}{l}\text { No of } \\
\text { staging } \\
\text { procedures }\end{array}$} \\
\hline & & & \multicolumn{2}{|l|}{ UJ13A } & \multicolumn{2}{|l|}{ Pool } & \\
\hline & Positive & Negative & Positive & Negative & Positive & Negative & \\
\hline $\begin{array}{l}4 \\
3 \\
2 \\
1\end{array}$ & $\begin{array}{r}10 \\
0 \\
1 \\
1\end{array}$ & $\begin{array}{r}8 \\
10 \\
1 \\
2\end{array}$ & $\begin{array}{l}6 \\
4 \\
0 \\
1\end{array}$ & $\begin{array}{r}10 \\
6 \\
2 \\
2\end{array}$ & $\begin{array}{l}5 \\
2 \\
0 \\
1\end{array}$ & $\begin{array}{r}11 \\
8 \\
2 \\
2\end{array}$ & $\begin{array}{c}18^{\star} \\
10 \\
2 \\
3\end{array}$ \\
\hline
\end{tabular}

${ }^{\star}$ No smears stored from two staging procedures, thus 16 sets of immunostained smears available from 18 staging procedures.

observer did not think tumour was present. If either observer could not commit himself, or there was disagreement, a combined review was undertaken at a double headed microscope. After completion of reporting and conferring the number code was broken and results correlated with individual patients, sites, and sampling dates.

The appearances of Romanowsky stained smears and trephine biopsy histological grades were taken from the original record made at the time of the staging procedure. Histological grading was as previously reported. ${ }^{1}$ Briefly, grade 1 described "normal" marrow, grade 2 marrow with increased reticulin as the only abnormality, grade 3 distorted architecture with increased fibrous tissue and grade 4 marrow with recognisable tumour cells.

Sixty internal experimental known control slides were used. "Known positive" controls using cytocentrifuge preparations from a stock supply of heavily infiltrated marrow and "known negative" controls using omission or substitution with mouse immunoglobulin of primary antibody, or using non-neuroblastoma material (acute leukaemia), were processed with each batch. In addition, 17 slides from patients without neuroblastoma, four from a child with stage II ganglioneuroblastoma, and four from a child with stage III neuroblastoma were numbered and inserted into the batches of test slides as blind controls.

Sets of results were grouped into staging procedures. In all cases the "worst" appearance of an individual test was taken as the final result of that staging procedure-for example, if a "positive" result were found in only one of four UJ13A tests in one staging procedure, the final result would be recorded as "positive". Results of routine cytological and histological examination were treated similarly.

\section{Results}

Three hundred and fifty one slides were examined. Ten were technically unsatisfactory and 60 were known internal controls. Twenty five were from patients who did not have disseminated neuroblastoma. Thus 256 slides from 33 staging procedures, two at diagnosis and 31 after treatment had started, in 12 patients were available for analysis.

Because of duplicate testing on some of the smears, the 256 slides comprised 152 tests with UJ13A and 153 with the "pool" of antibodies. Of these 305 antibody tests, 244 were carried out on marrow smears and 61 on cytocentrifuged collagenase digests.

Concordant results between the two observers were obtained in 235 of 305 (77\%) tests. Disagreement was exceptional, but most of the $70(23 \%)$ results requiring conferring were "equivocal" due to the presence of immunostained cells which could not confidently be identified as either normal marrow constituents or tumour.

One of the 25 blind control slides from patients without disseminated neuroblastoma was recorded as positive with UJ13A alone. This patient had stage II ganglioneuroblastoma and was treated with surgery alone without subsequent relapse to date. All others were reported as negative.

Table 1 shows the relation between results of UJ13A and pool tests on smears from each staging procedure, together with Romanowsky stained smear results, and the corresponding trephine histological grade. Table 2 shows the cytocentrifuged collagenase digest immunocytochemistry results related to histological grade, and table 3 the relation between routine cytological examination and antibody tests.

Antibody tests did not always confirm the presence of tumour on smears when the histological appearances were grade 4 (tumour cells identifiable), nor did routine cytological examination of the smears. Grade 3 histology (distorted architecture and fibrosis) was associated with positive antibody tests in four of 10 sets of marrow smears and one of three sets of collagenase digests, but with none of 10 sets of Romanowsky stained smears. Although most Romanowsky negative staging procedures were also antibody negative, the

Table 2 Immunocytochemical staining of cytocentrifuged collagenase digests compared with trephine biopsy histology

\begin{tabular}{|c|c|c|c|c|c|}
\hline \multirow[b]{3}{*}{ Trephine histological grade } & \multicolumn{4}{|c|}{ Cytocentrifuge digest immunocytochemistry } & \multirow[b]{3}{*}{ No of staging procedures ${ }^{\star}$} \\
\hline & \multicolumn{2}{|l|}{$U J 13 A$} & \multicolumn{2}{|l|}{ Pool } & \\
\hline & Positive & Negative & Positive & Negative & \\
\hline $\begin{array}{l}4 \\
3 \\
2 \\
1\end{array}$ & $\begin{array}{l}4 \\
1 \\
0 \\
0\end{array}$ & $\begin{array}{l}2 \\
2 \\
0 \\
1\end{array}$ & $\begin{array}{l}5 \\
1 \\
0 \\
0\end{array}$ & $\begin{array}{l}1 \\
2 \\
0 \\
1\end{array}$ & $\begin{array}{l}6 \\
3 \\
0 \\
1\end{array}$ \\
\hline
\end{tabular}

«No digests available from 23 staging procedures. 
Table 3 Immunocytochemistry compared with Romanowsky staining of marrow smears

\begin{tabular}{|c|c|c|c|c|c|}
\hline \multirow[b]{3}{*}{ Romanowsky staining } & \multicolumn{4}{|c|}{ Immunocytochemistry } & \multirow[b]{3}{*}{ No of staging procedures } \\
\hline & \multicolumn{2}{|l|}{$U J 13 A$} & \multicolumn{2}{|l|}{ Pool } & \\
\hline & Positive & Negative & Positive & Negative & \\
\hline $\begin{array}{l}\text { Positive } \\
\text { Negative }\end{array}$ & $\begin{array}{l}6 \\
5\end{array}$ & $\begin{array}{r}4 \\
16\end{array}$ & $\begin{array}{l}5 \\
2\end{array}$ & $\begin{array}{r}5 \\
19\end{array}$ & $\begin{array}{l}10 \\
21\end{array}$ \\
\hline
\end{tabular}

«No smear immunocytochemistry from two staging procedures, thus a total of 31 staging procedures with both Romanowsky and immunostaining available.

correlation between antibody tests and routine cytology was poor. In summary, immunostaining suggested the presence of tumour in seven of 18 staging procedures in which conventional histological or cytological investigations had shown infiltration. Tumour infiltration was also suggested in four of 10 staging procedures with suspicious grade 3 histological features, but in none of three staging procedures with relatively innocent histological and cytological appearances.

Numerous possibilities for false positive reactions were discovered. The reactivity of the monoclonal antibodies used is not restricted to neuroectodermal cells. Occasional positive cells were found even in normal marrow. In particular, three types of positive cell were detected which did not convince either observer that they represented tumour. The first of these were small, very brightly staining cells, often with a small trail of cytoplasm (fig 1). Secondly, larger single "macrophage-

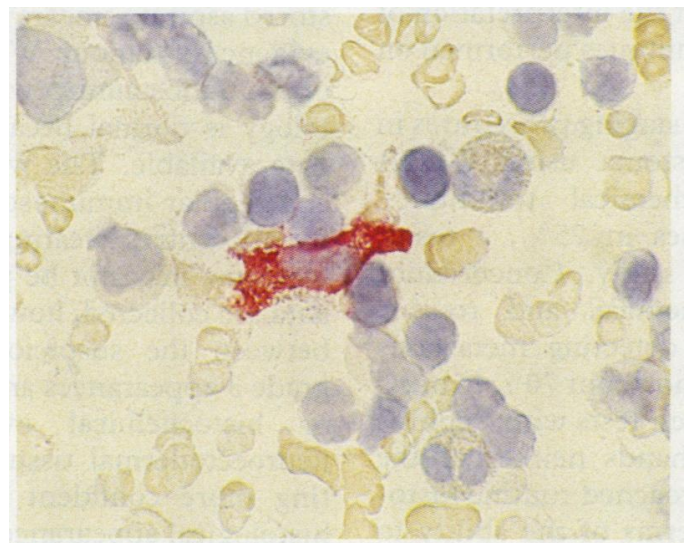

Figure 1 Example of small brightly staining cells found in normal and infiltrated marrow. (Immunoalkaline phosphatase/UJ13A.)

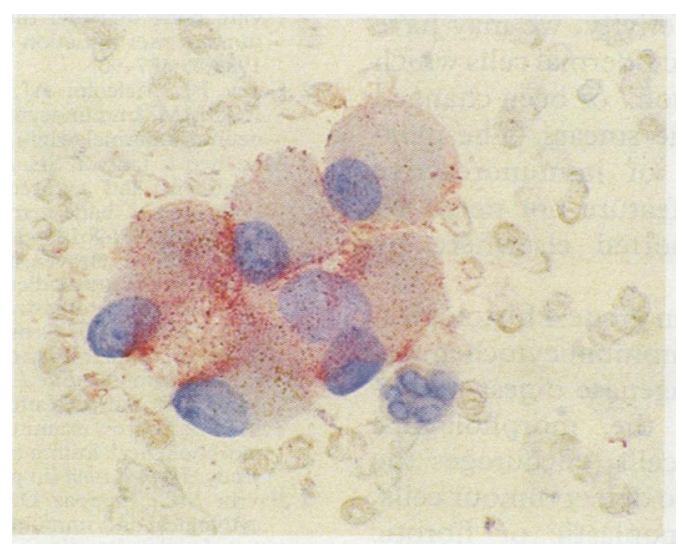

Figure 3 Immunostaining osteoblasts. (Immunoalkaline phosphatase/UJ13A.) like" cells with oval nuclei and irregular trailing cytoplasm, only very rarely found in normal marrow, were frequently present in smears from the children with neuroblastoma (fig 2). Finally, osteoblasts often stained brightly, especially at the periphery of the cell (fig 3). They appeared both singly and in clumps and formed the only readily identifiable group of non-malignant cells which reacted with the monoclonal antibodies in this study. Their morphological appearance is quite distinct from that of clumps of tumour cells (fig 4).

\section{Discussion}

The ability to perform immunofluorescence or immunocytochemical tests is within the capacity of most haematology and pathology departments, but their interpretation is by no means easy. We found that immunofluorescence techniques for detecting neuroblastoma cells in aspirated bone marrow were unsatisfac-

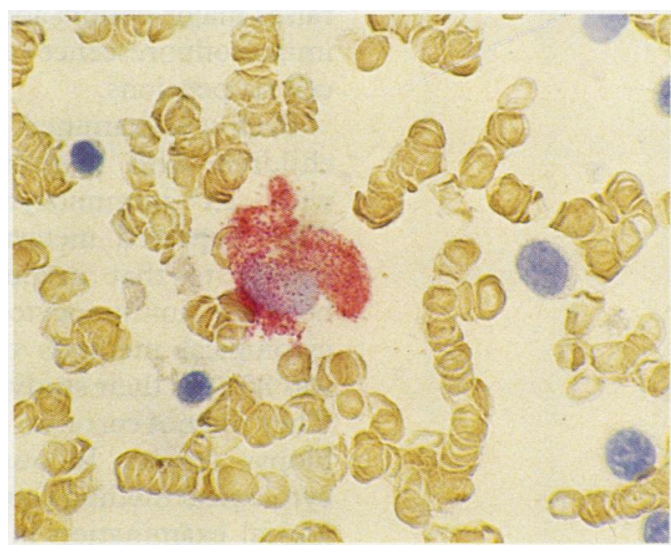

Figure 2 Immunostaining "macrophage-like" cell. These were found occasionally in normal marrow, and more frequently in infiltrated marrow. Their presence alone did not confer positivity for tumour infiltration in this study. (Immunoalkaline phosphatase/UJ13A.)

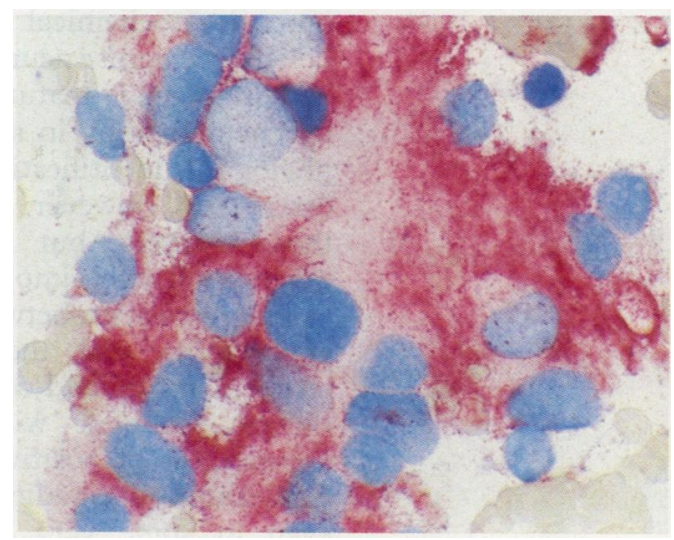

Figure 4 Clump of immunostaining tumour cells. (Immunoalkaline phosphatase/UJ13A.) 
tory because only the first few drops of aspirate were likely to provide diagnostic features; subsequent aspiration resulted in dilution with blood, and clumps of tumour cells did not survive the separation and washing procedures. We therefore chose an immunocytochemical method applied to smears of native marrow to show reactivity with the monoclonal antibodies in our study. The technique also permits reexamination of the morphology of reacting cells rather than relying on the ephemeral nature of fluorescence.

Rogers et al reported a high sensitivity and specificity for UJ13A in the detection of neuroblastoma in aspirated marrow by immunofluorescence, but used necessarily arbitrary criteria for defining the "true" answers against which their tests are judged. ${ }^{4}$ This is a difficulty in the assessment of any of these immunological techniques. The only results which can be said to represent the "true" answer are the positive identification of tumour cells on Romanowsky stained smears and biopsy specimens, but even these remain, to some extent, a matter of judgment. Negative immunological results cannot be equated with absence of metastases. UJ13A negative tumour cells were found in several of our patients at diagnosis, intermingled with positive cells. Positive results do not by themselves constitute proof of infiltration. The variety of cells, whether single or clumped, which reacted with UJ13A and the other antibodies in this study, raises major doubts about the interpretation of immunofluorescence techniques performed on cell suspensions.

Beck et al examined 75 staging procedures in children with neuroblastoma using UJ13A with an immunocytochemical technique. ${ }^{5}$ They reported metastases in $25 \%$, a result similar to that in our study. Concordance between immunocytochemical and routine cytological methods of detecting metastases was $80 \%$ in their study and about $70 \%$ in ours. They did not correlate their tests with trephine biopsy results. In our hands neither of the cytological methods approached routine histological examination in terms of the ability to detect tumour.

We are not as confident as Beck et alt of our ability to exclude false positive cells such as macrophages. Although not convincing as tumour and reported as negative despite immunocytochemical reactivity, we may have failed to recognise neuroectodermal cells which had undergone "maturation" or been changed by chemotherapy in some smears. The morphological identification of immunoreactive osteoblasts, however, a feature not noted by those workers, but reported elsewhere by others $^{6}$ is usually simple.

The correlation between grade 4 histological appearances and the immunocytochemical tests on smears and collagenase digest preparations, coupled with the morphological appearances of positive cells, encourages the view that the antibodies do detect tumour cells, even in some very hypoplastic or fibrotic marrows. Despite the caveats outlined above, this study strengthens the suggestion made earlier ${ }^{1}$ that grade 3 histological appearances (abnormal architecture and fibrosis) imply persisting neuroectodermal tissue. The ability of collagenase digestion to liberate cells from biopsy cores without destroying the relevant antigens encourages further use of this technique.

There was good correlation between UJ13A and the pool of antibodies in this study, but it is not large enough to detect loss of reactivity of tumour cells to one or more antibodies and does not provide data concerning the necessity for several antibodies.

As noted by Beck et $a l^{5}$ and Favrot $e t a l,{ }^{7}$ this study has shown that immunological investigations cannot replace standard light microscopical examination of smears and, in particular, biopsy cores, but that they do provide complementary information. The degree to which a positive immunological test should influence a staging evaluation must remain for the moment a matter of debate. The ability to re-examine material together with the acquisition of experience may yet indicate the proper place for immunological investigations of potentially infiltrated marrow in this disease.

We believe that if tumour is detectable in representative, good quality biopsy cores, immunostaining of aspirates does not provide additional useful information about the presence of infiltration. When grade 3 histological features are found, immunostaining of stored aspirate smears may provide convincing evidence of tumour. We cannot assess the value of immunostaining when bone marrow histology is normal because of the very limited data available. The workload is considerable and regular immunostaining of every aspirate by all centres treating children with neuroblastoma may not be practicable. If sufficient data are collected, however, a close association between the suspicious but not diagnostic grade 3 appearances and immunocytochemical or histochemical evidence of persisting neuroectodermal tissue may emerge, permitting more confident interpretation of these histological appearances by centres that do not carry out regular immunological investigations.

1 Reid MM, Hamilton PJ. Histology of neuroblastoma involving bone marrow: the problem of detecting residual tumour after initiation of chemotherapy. Br J Haematol 1988;40:487-90.

2 Carey PJ, Malcolm AJ, Pearson ADJ, Kemshead JT, Reid MM. Immunocytochemical detection of tumours of neuroectodermal origin. J Clin Pathol 1988;41:586-9.

3 Mononen I, Jansson SE. A rapid procedure by collagenase treatment and cytocentrifugation for the cytological evaluation of bone marrow biopsy specimens. Clin Lab Haematol 1986;8:149-54.

4 Rogers DW, Treleaven JG, Kemshead JT, Pritchard J. Monoclonal antibodies for detecting bone marrow invasion by neuroblastoma. J Clin Pathol 1989;42:422-6.

5 Beck D, Maritaz D, Gross N, et al. Immunocytochemical detection of neuroblastoma cells infiltrating clinical bone detection of neuroblastoma cells infiltrating clinical

6 Oppedal BR, Storm Mathisen I, Kemshead JT, Brandtzaeg P. Bone marrow examination in neuroblastoma patients; a P. Bone marrow examination in neuroblastoma patients; a morphological immunocyto-

7 Favrot MC, Frappaz D, Maritaz O, et al. Histological, cytological and immunological analyses are complementary for the detection of neuroblastoma cells in the bone marrow. Br J Cancer 1986;54:637-41. 